\title{
A influência de uma universidade na geração de um polo regional de ensino superior
}

\begin{abstract}
Moacir Luiz Righi Graduação em Ciências Contábeis pela Universidade Federal de Santa Maria -

UFSM

Contador da Universidade Federal de Santa Maria - UFSM Centro de Ciências Rurais, prédio 97. Santa Maria/RS. CEP: 97105-900

E-mail: righilbj@hotmail.com

Janis Elisa Ruppenthal

Doutorado em Engenharia de Produção pela Universidade Federal de Santa Catarina -

UFSC

Professora da Universidade Federal de Santa Maria Avenida Roraima, № 1.000. Camobi. Santa Maria/RS. CEP: 97105-900

E-mail: janis.rs.br@gmail.com
\end{abstract}

\section{RESUMO}

O ensino superior tem apresentado um crescimento surpreendente nos últimos anos no Brasil. Com esse crescimento, as universidades públicas tornam-se referência de ensino e de atração de estudantes para as cidades onde se localizam, e muitas vezes essas instituições não conseguem atender a demanda de todos os estudantes. Assim, nascem novas instituições formadas para atrair estudantes de áreas e cursos não oferecidos pelas universidades públicas ou para suprir a procura por vagas não preenchidas por essas universidades. Esse artigo tem por objetivo mostrar a contribuição da Universidade Federal de Santa Maria - UFSM na criação de um polo de ensino superior em Santa Maria - RS. Para isso, se avalia estatisticamente o número de cursos de graduação presenciais, de alunos e de professores desses cursos, para confirmar a existência de uma correlação entre as variáveis estudadas. Os resultados mostram que a UFSM contribuiu no desenvolvimento de um polo de ensino superior no município de Santa Maria, concluindo-se pela importância da cidade contar com uma instituição de ensino superior pública.

Palavras-chave: Polo de ensino superior. UFSM. Desenvolvimento.

The influence of a university in the generation of a regional pole of higher education

\section{ABSTRACT}

Higher education has shown a surprising growth in recent years in Brazil. With this growth, public universities become teaching reference and attract students to the cities 
where they are located. Sometimes these institutions cannot attend the demand of all students. Thus we find new institutions formed to attract students and courses in areas not offered by public universities or to meet the unmet demand by vague by public universities. This paper aims to show the influence of the Federal University of Santa Maria - UFSM in creating a pole of higher education in Santa Maria. For this, assesses statistically the number of undergraduate classroom, students and professors of these courses, to confirm the existence of a correlation between variables. The results show that the UFSM was influential in the development of a center of higher education in the town of Santa Maria, concluding the importance of a public institution of higher education to the cities.

Keywords: Polo higher education. UFSM. Development.

\section{INTRODUÇÃO}

As universidades têm se constituído em peça-chave do processo de desenvolvimento regional. Essas instituições possuem missão de gerar e transmitir conhecimento, formar capital intelectual, incentivar as inovações e promover a transferência de tecnologias. Com isso, desencadeia-se um processo de mudança econômico-social através da criação de um ambiente intelectualmente diferenciado, o qual facilita o processo de transformação das estruturas produtivas das regiões onde estão localizadas.

A análise da contribuição das universidades para o desenvolvimento regional tem adquirido relevância nos últimos cinco anos, principalmente com o plano de expansão do governo federal para o ensino de nível superior. Em geral, essa análise é realizada por meio de duas perspectivas: a primeira, atentando para o impacto da universidade em termos de geração de conhecimento, cujos efeitos se materializam em médio e longo prazo; e a segunda, definindo a universidade como um agente econômico, ou seja, proprietária de um orçamento e executora de despesas.

As instituições de ensino superior distinguem-se em função de sua localização. Muitas universidades localizadas em cidades mais importantes regionalmente destacam-se pela extensão dos complexos construídos, grandes orçamentos para manutenção, investimentos e gastos com pessoal. Há evidências que os investimentos 
governamentais em educação retornam em médio e longo prazo em forma de desenvolvimento e crescimento econômico (FELSENSTEIN, 1996; GARRIDO-YSERTE E GALLO-RIVERA, 2010).

Os impactos gerados pela implantação de uma universidade em uma região ocorrem nas áreas: demográfica, política, econômica, de infraestrutura, cultural, educacional, de atração de investimentos e de aspectos sociais. Esses efeitos relacionam-se direta ou indiretamente e sofrem a influência do nível de desenvolvimento e crescimento das instituições (GARRIDO-YSERTE E GALLORIVERA, 2010; HOFF et al., 2011).

Considerando o exposto, o objetivo desse artigo é avaliar a contribuição da UFSM na criação de um polo de ensino superior em Santa Maria. Para tanto, são apresentados dados coletados por meio de levantamentos e pesquisa bibliográfica, a partir dos quais é analisada a contribuição da Universidade Federal de Santa Maria UFSM na geração e desenvolvimento de um polo regional de ensino superior.

A escolha desse tema tem como finalidade contribuir para elucidar a importância e o significado das universidades públicas para o dinamismo e criação de polos de ensino superior nas regiões onde estão localizadas.

O artigo divide-se em seções, sendo na seção 2 apresentado o impacto das universidades no desenvolvimento regional. Na seção 3 é apresentada a metodologia utilizada na condução desse estudo. A seção 4 apresenta o estudo do caso da UFSM, e a sua contribuição no processo de desenvolvimento de um polo de ensino superior na cidade de Santa Maria. Por fim são apresentadas as considerações finais do estudo, seguidas das referências.

\subsection{0 impacto das universidades no desenvolvimento regional}

A universidade, através de sua função de ensino, pesquisa e extensão, desempenha papel de destaque na formação e disseminação de conhecimento, promovendo o desenvolvimento econômico-social da região. Sua atuação junto ao setor produtivo cumpre funções e tarefas diversificadas, como: disponibilização de suporte 
científico e tecnológico, formação de recursos humanos, desenvolvimento de pesquisas e tecnologias para serem utilizadas na própria região ou com aplicação comercial. Isso permite que o setor enfrente um ambiente de alta competitividade (BOVO, 2008).

A implantação de uma universidade pública transforma as economias regionais, pois gera impactos diretos e indiretos. Os impactos diretos se relacionam com a movimentação de recursos financeiros que ocorrem por meio do pagamento de salários aos professores e funcionários, investimentos em obras e equipamentos, demais despesas de custeio e gastos dos alunos oriundos de outros municípios, que dão origem a variados efeitos multiplicadores que se auto-reforçam e se propagam de maneira cumulativa. Essa movimentação de recursos produz impactos econômicos e financeiros que ganham maior significado quando inseridos no contexto da economia dos municípios onde as universidades estão inseridas. Como impactos indiretos podem ser citados os serviços oferecidos pelas universidades, e que se traduzem em economia para os poderes públicos. O caso mais paradigmático da economia de recursos resultante da redução da aquisição de serviços de educação superior referese às atividades de ensino. É o caso dos alunos que, dada a oportunidade de estudarem na universidade local, não se deslocam para outros municípios.

Com isso, os recursos que manteriam esses alunos em outras cidades permanecem na economia local. Além disso, muitos empregos indiretos são gerados em diferentes setores, decorrentes da dinamização da economia local e, principalmente, da contratação de serviços pela universidade, como os serviços de segurança e de limpeza. Esses benefícios são obtidos, primeiramente, porque nessas localidades é que os resultados das ações, pesquisas e programas realizados pelas instituições são mais percebidos. Segundo, porque as economias locais são diretamente beneficiadas pelas despesas diretas e indiretas relacionadas ao funcionamento dessas instituições (WARREN et al., 2008).

Para Drucker e Goldstein (2007), quando o capital humano, as pesquisas e outros recursos de capital são alocados juntos em uma mesma região, produz-se uma concentração de auto-reforço e gera-se o poder de estimular o crescimento econômico 
dessa região. Para Goldstein e Glaser (2012), as regiões que possuem maiores ativos de conhecimento tendem a destacar-se nesse momento de economia globalizada. A influência das universidades, nesse caso, é observada pelo conhecimento gerado, pela liderança de pessoas que fazem parte do quadro funcional da instituição e da própria universidade sobre a comunidade onde está instalada. Todos esses fatores ligados às universidades auxiliam na resolução de problemas, fomentando o crescimento regional.

Steinacker (2005) considera que além das universidades de grande porte, como as públicas, o impacto de pequenas instituições também é relevante regionalmente. Rolim e Kureski (2007); Rolim e Serra (2009) e Hoff et al. (2011) apontam em seus estudos as influências que as universidades desencadeiam nas regiões onde se instalam, desde o aumento populacional, passando pelo aquecimento imobiliário, atividades comerciais e prestação de serviços. Para Siegfried et al. (2007), relevantes estudos tem sido realizados por diferentes pesquisadores desde 1971, voltados para a avaliação dos impactos econômicos das universidades sobre suas regiões, mostrando a geração direta de empregos, o impacto dos investimentos na economia local e a participação na composição da força de trabalho local.

De acordo com Drucker e Goldstein (2007), pode se observar cinco abordagens importantes tomadas na literatura para análise e avaliação do impacto das universidades no desenvolvimento regional: estudos de uma universidade isolada; pesquisas ou questionários; estimativas de função produção; estimativas de funções transversais; e projetos de impacto no estilo quasiexperimental. Além dessas abordagens, outra se destaca pelo tratamento diferenciado ao assunto: é o conceito de universidade-produto abordado por Lendel (2010).

Goldstein e Renault (2005) e Lendel (2010) identificaram oito diferentes funções, ou saídas, das universidades modernas, que podem influenciar no desenvolvimento econômico local: criação de conhecimento; a criação de capital humano; a transferência de know-how existente; tecnologia; investimentos; liderança regional; conhecimento de infraestrutura de produção; e influência sobre milieu regional. 
A formulação, a partilha de conhecimento e a sabedoria têm sido a razão de existência das universidades desde suas origens na Europa na idade média. A criação do capital humano é o segundo componente da missão dessas instituições. O desenvolvimento do capital humano é intrínseco ao processo de estabelecimento de novos conhecimentos, de professores, estudantes e pesquisadores, para desenvolverem habilidades técnicas e intelectuais. Isso ocorre, também, através de atividades como ensino à distância, programas de extensão e programas de educação comunitária. A transferência de tecnologia e know-how é análoga à criação de capital humano, mas centra-se na aplicação dos conhecimentos existentes para resolver um problema específico, normalmente melhorar produtos ou aperfeiçoar processos (LENDEL, 2010).

As universidades são também importantes atores regionais para o volume de investimento em capital físico: construção e manutenção de edifícios, laboratórios, parques tecnológicos e outros tipos de instalações, juntamente com transporte associado e outras infraestruturas.

Para Lendel (2010), o conceito de infraestrutura de conhecimento tornou-se familiar através da literatura de economia com a teoria de crescimento endógeno, como um componente de um conceito mais amplo de economias de aglomeração regional. A infraestrutura de conhecimento pode ser definida como a geração e armazenagem de conhecimento, juntamente com os componentes institucionais e organizacionais que suportam o crescimento e o aplicativo. Fava-de-Moraes (2000) salienta a participação da inovação e do financiamento de universidades com recursos provenientes de empresas privadas, como diferencial para o aumento e atualização tecnológica existente nos Estados Unidos da América, como forte incentivo à pesquisa básica.

Finalmente, a noção de uma influência de universidade no milieu regional abrange a gama de contribuições distintivas que as universidades oferecem aos seus arredores, sejam elas intelectuais, sociais, culturais ou recreativos, atraindo uma concentração de profissionais altamente qualificados e criativos, e estabelecendo uma determinada localização dinâmica (LENDEL, 2010; GOLDSTEIN; GLASER, 2012). 
Cronologicamente, alguns estudos realizados no Brasil sobre o impacto de uma universidade sobre a economia são os de Bastos (1987), que pesquisou o sistema de custos para Universidades Federais. O estudo de Schwartzman (1995) estabeleceu uma metodologia para ordenação das universidades brasileiras, através da construção de indicadores que reflitam aspectos qualitativos e quantitativos do processo produtivo das mesmas. Marinho (1996) realiza um estudo sobre a avaliação organizacional de uma universidade pública envolvendo a eficiência na gestão dos recursos. Mais recentemente, buscando inspiração na literatura estrangeira, podem ser destacados, os estudos de Bovo (2008); Rolim e Kureski (2010) e Gubiani et al. (2010).

Bovo (2008) procurou dimensionar o montante de recursos monetários movimentados pelas unidades universitárias da UNESP. O estudo buscou avaliar os impactos econômicos e financeiros da circulação desses recursos para os municípios onde os campi da universidade estão instalados. Utilizando-se também de questionários, Bovo (2008) observou a média de gastos de alunos oriundos de outras cidades, bem como a média de gastos dos servidores na própria cidade.

Rolim e Kureski (2010) utilizaram as técnicas de insumo-produto. O estudo considera o impacto de todo o sistema federal de ensino superior na economia brasileira. Assim, foram considerados os gastos do sistema, nas diferentes rubricas, a partir dos microdados do Censo do Ensino Superior de 2005 realizado pelo INEP/MEC. Além desses dados, foi utilizada a nova matriz de insumo-produto de 2005 do IBGE. Com esse conjunto de dados foi possível avaliar os impactos e os seus efeitos multiplicadores sobre toda a economia nacional.

Gubiani et al. (2010) propõem um modelo para medição das atividades desenvolvidas na dimensão das universidades, analisando os impactos na dimensão da economia através da análise de variáveis, indicadores e índices como o Índice de Desenvolvimento Humano dos Municípios (IDH-M). 


\section{METODOLOGIA}

A maior parte das pesquisas, inclusive as citadas anteriormente, aborda a importância das universidades para o desenvolvimento econômico das regiões. Dessa forma, em razão de existirem poucos estudos sobre a contribuição dessas instituições na formação do capital intelectual, esse artigo direciona o estudo para esse aspecto. Assim, partindo-se da criação da UFSM, avaliou-se sua contribuição na criação e no desenvolvimento de um polo de ensino superior em Santa Maria.

Com esse objetivo, partiu-se para a coleta de dados primários e secundários sobre o número de cursos de graduação, número de alunos matriculados e formados, número de técnico-administrativos e de docentes das sete instituições de ensino superior instaladas em Santa Maria. Malhotra (2006) classifica os dados primários como os gerados pelo pesquisador, com a finalidade de solucionar seu problema de pesquisa. Os dados secundários são encontrados em relatórios, boletins e pesquisas de outros. Assim, a coleta de dados foi realizada em duas etapas. Os dados secundários foram obtidos em anuários, livros e sites sobre os indicadores da UFSM. Os dados primários foram obtidos mediante a utilização da técnica de levantamento. Essa etapa foi realizada com o auxílio de questionários desenvolvidos para coletar os dados das instituições privadas de ensino superior de Santa Maria.

Para desenvolver esse estudo foram avaliadas as variáveis nas instituições com ensino superior presencial, envolvendo somente os cursos de graduação, por ser um nível de ensino comum às sete instituições pesquisadas. Essa é a limitação dessa pesquisa, visto que nem todas as instituições pesquisadas disponibilizam cursos de pós-graduação lato-sensu e stricto-sensu, bem como nem todas oferecem a modalidade de ensino à distância. Assim, essa pesquisa parte do centro de estudo, a UFSM e, a partir dessa instituição estabelece uma relação com a evolução do ensino superior em Santa Maria. As inter-relações entre esses elementos são mostradas na Figura 1. 
A etapa de levantamentos foi realizada nas seguintes instituições: UNIFRA Centro Universitário Franciscano, FAPAS - Faculdade Palotina de Santa Maria, FADISMA - Faculdade de Direito de Santa Maria, ULBRA - Universidade Luterana do Brasil, FISMA - Faculdade Integrada de Santa Maria e FAMES - Faculdade Metodista de Santa Maria.

Após a coleta e registro dos dados em planilhas eletrônicas, esses foram analisados e interpretados. A análise de dados quantificados sumariza a apresentação dos mesmos, auxiliando os pesquisadores na resolução do problema da pesquisa. A interpretação desses dados fortalece as conclusões acerca dessas respostas, relacionando os dados a conhecimentos prévios.

- Para a elaboração do artigo foi proposta a questão de pesquisa: "Qual a contribuição da UFSM na criação de um polo de ensino superior na cidade de Santa Maria?"

- A UFSM contribuiu para o desenvolvimento de umpolo de ensino superiorna cidade de Santa Maria

- Para cumprir com os objetivos, o plano tratou a pesquisa como exploratória, na qual os dados sãotratados estatiscamentepara serem analisados e comparados

- As v ariáv eis consideradas foram o número de cursos de graduação, o número de alunos matriculados e formados e o número de docentes e técnico-administrativ os, além de dados orçamentários

- O questionário inicial continha seis questões sobre as variáveis iniciais consideradas

- Durante a realização do pré-teste, foi descartada a questão relacionada à parte orçamentária, visto que os participantes não Pré-test concordaram em responder ao questionamento

- Para ter confiança estatística, foram pesquisadas todas as instituições priv adas de ensino superior presencial da cidade de Santa Maria

- A coleta de dados foi realizada por meio de questionários ap licados às instituições particulares e através de pesquisa bibliográfica para os dados da UFSM

- A análise dos dados foi realizada com o auxílio do software "Statistica 9.0 ("

- Por fim, foi realizada a interpretação dos dados, para a confiramação da hipótese e conclusão do estudo

Figura 1: Inter-relação entre elementos da pesquisa e método de condução

Fonte: elaborado pelos autores. 
Foi realizada uma análise descritiva entre as variáveis pesquisadas da UFSM e das demais instituições de ensino superior presencial de Santa Maria. Foram realizadas análises com base no coeficiente de correlação linear de postos ou de Spearman, que é uma medida não paramétrica e destina-se a determinar o grau de associação entre duas variáveis com o objetivo de estudar a correlação entre duas classificações.

Foi considerado um nível de $5 \%$ de significância e as análises estatísticas foram realizadas com auxílio do software "Statistica 9.0 ®", comparando individualmente os dados da UFSM com cada uma das demais instituições. Após essa análise, foi realizada a avaliação da contribuição da UFSM no desenvolvimento de um polo de ensino superior em Santa Maria.

\section{FORMAÇÃO DE UM POLO DE ENSINO SUPERIOR EM SANTA MARIA}

As primeiras questões do questionário buscavam conhecer o histórico das instituições em relação ao início de suas atividades e à escolha dos cursos de graduação ofertados. Para tanto foram apresentadas aos seus gestores duas questões fechadas. A primeira questão interrogava sobre os principais motivos da escolha pela cidade de Santa Maria para a instalação da instituição, cujo resultado é mostrado na Tabela 1.

Tabela 1: Motivos da instalação das instituições na cidade de Santa Maria

\begin{tabular}{|c|c|}
\hline Motivo & Percentual \\
\hline Santa Maria possui polo de ensino superior & $66 \%$ \\
\hline Número de alunos preparando-se para o vestibular da UFSM & $17 \%$ \\
\hline Posicionamento geográfico da cidade & $17 \%$ \\
\hline
\end{tabular}

Fonte: elaborado pelos autores com base nos dados da pesquisa.

Observa-se na Tabela 1 que a maioria dos gestores das instituições pesquisadas (66\%) considera a existência de um polo de ensino superior, o qual favorece a criação e o desenvolvimento de instituições de ensino superior. Esse desenvolvimento pode estar 
relacionado com alguns fatores considerados nas instituições participantes da pesquisa, conforme dados obtidos nos questionários, como pode ser observado na Tabela 2.

Tabela 2: Motivos da escolha de cursos ofertados pelas instituições na cidade de Santa Maria

\begin{tabular}{|c|c|}
\hline Motivo & Percentual \\
\hline Ofertar cursos com demanda reprimida em Santa Maria & $17 \%$ \\
\hline Ofertar novos cursos ainda não oferecidos pelas outras instituições de ensino superior & $17 \%$ \\
\hline Surgimento de novas áreas de ensino e tecnologias & $66 \%$ \\
\hline
\end{tabular}

Fonte: elaborado pelos autores com base nos dados da pesquisa.

Observa-se que $66 \%$ da oferta de novos cursos de graduação pelas instituições de ensino superior de Santa Maria é determinada pelo surgimento de novas áreas de ensino e tecnologias. Isso faz com que as instituições mantenham-se atualizadas para suprir as demandas por profissionais qualificados, capazes de suprir as necessidades das organizações.

\subsection{Números da graduação presencial em Santa Maria}

A Tabela 3 mostra a evolução no número de cursos de graduação presencial das instituições de ensino superior da cidade de Santa Maria. 
Tabela 3: Número de cursos de graduação presencial nas instituições da cidade de Santa Maria

\begin{tabular}{|c|c|c|c|c|c|c|c|c|}
\hline Ano & UFSM & UNIFRA & ULBRA & FAMES & FADISMA & FAPAS & FISMA & TOTAL \\
\hline 1997 & $\begin{array}{l}53 \\
(85,5 \%)\end{array}$ & $\begin{array}{l}9 \\
(14,5 \%)\end{array}$ & & & & & & 62 \\
\hline 1998 & $\begin{array}{l}54 \\
(81,82 \%)\end{array}$ & $\begin{array}{l}10 \\
(15,15 \%)\end{array}$ & & $\begin{array}{l}2 \\
(3,03 \%)\end{array}$ & & & & 66 \\
\hline 1999 & $\begin{array}{l}55 \\
(73,33 \%)\end{array}$ & $\begin{array}{l}18 \\
(24,00 \%)\end{array}$ & & $\begin{array}{l}2 \\
(2,67 \%)\end{array}$ & & & & 75 \\
\hline 2000 & $\begin{array}{l}56 \\
(70,00 \%)\end{array}$ & $\begin{array}{l}20 \\
(25,00 \%)\end{array}$ & & $\begin{array}{l}2 \\
(2,50 \%)\end{array}$ & & $\begin{array}{l}2 \\
(2,50 \%)\end{array}$ & & 80 \\
\hline 2001 & $\begin{array}{l}56 \\
(69,14 \%)\end{array}$ & $\begin{array}{l}21 \\
(25,93 \%)\end{array}$ & & $\begin{array}{l}2 \\
(2,47 \%)\end{array}$ & & $\begin{array}{l}2 \\
(2,47 \%)\end{array}$ & & 81 \\
\hline 2002 & $\begin{array}{l}56 \\
(63,64 \%)\end{array}$ & $\begin{array}{l}23 \\
(26,14 \%)\end{array}$ & $\begin{array}{l}5 \\
(5,68 \%)\end{array}$ & $\begin{array}{l}2 \\
(2,27 \%)\end{array}$ & & $\begin{array}{l}2 \\
(2,27 \%)\end{array}$ & & 88 \\
\hline 2003 & $\begin{array}{l}55 \\
(59,14 \%)\end{array}$ & $\begin{array}{l}26 \\
(27,96 \%)\end{array}$ & $\begin{array}{l}5 \\
(5,38 \%)\end{array}$ & $\begin{array}{l}5 \\
(5,38 \%)\end{array}$ & & $\begin{array}{l}2 \\
(2,15 \%)\end{array}$ & & 93 \\
\hline 2004 & $\begin{array}{l}56 \\
(56,57 \%) \\
\end{array}$ & $\begin{array}{l}27 \\
(27,27 \%)\end{array}$ & $\begin{array}{l}5 \\
(5,05 \%) \\
\end{array}$ & $\begin{array}{l}6 \\
(6,06 \%) \\
\end{array}$ & $\begin{array}{l}1 \\
(1,01 \%)\end{array}$ & $\begin{array}{l}2 \\
(2,02 \%) \\
\end{array}$ & $\begin{array}{l}2 \\
(2,02 \%) \\
\end{array}$ & 99 \\
\hline 2005 & $\begin{array}{l}63 \\
(57,27 \%) \\
\end{array}$ & $\begin{array}{l}28 \\
(25,45 \%)\end{array}$ & $\begin{array}{l}7 \\
(6,36 \%)\end{array}$ & $\begin{array}{l}7 \\
(6,36 \%)\end{array}$ & $\begin{array}{l}1 \\
(0,91 \%)\end{array}$ & $\begin{array}{l}2 \\
(1,82 \%)\end{array}$ & $\begin{array}{l}2 \\
(1,82 \%)\end{array}$ & 110 \\
\hline 2006 & $\begin{array}{l}68 \\
(56,67 \%)\end{array}$ & $\begin{array}{l}29 \\
(24,17 \%)\end{array}$ & $\begin{array}{l}8 \\
(6,67 \%)\end{array}$ & $\begin{array}{l}7 \\
(5,83 \%)\end{array}$ & $\begin{array}{l}1 \\
(0,83 \%)\end{array}$ & $\begin{array}{l}4 \\
(3,33 \%)\end{array}$ & $\begin{array}{l}3 \\
(2,50 \%)\end{array}$ & 120 \\
\hline 2007 & $\begin{array}{l}67 \\
(55,83 \%)\end{array}$ & $\begin{array}{l}30 \\
(25,00 \%)\end{array}$ & $\begin{array}{l}8 \\
(6,67 \%)\end{array}$ & $\begin{array}{l}7 \\
(5,83 \%)\end{array}$ & $\begin{array}{l}1 \\
(0,83 \%)\end{array}$ & $\begin{array}{l}4 \\
(3,33 \%)\end{array}$ & $\begin{array}{l}3 \\
(2,50 \%)\end{array}$ & 120 \\
\hline 2008 & $\begin{array}{l}66 \\
(55,46 \%)\end{array}$ & $\begin{array}{l}30 \\
(25,21 \%)\end{array}$ & $\begin{array}{l}8 \\
(6,72 \%)\end{array}$ & $\begin{array}{l}7 \\
(5,88 \%)\end{array}$ & $\begin{array}{l}1 \\
(0,84 \%)\end{array}$ & $\begin{array}{l}4 \\
(3,36 \%)\end{array}$ & $\begin{array}{l}3 \\
(2,52 \%)\end{array}$ & 119 \\
\hline 2009 & $\begin{array}{l}94 \\
(63,51 \%)\end{array}$ & $\begin{array}{l}31 \\
(20,95 \%)\end{array}$ & $\begin{array}{l}8 \\
(5,41 \%)\end{array}$ & $\begin{array}{l}7 \\
(4,73 \%)\end{array}$ & $\begin{array}{l}1 \\
(0,68 \%)\end{array}$ & $\begin{array}{l}4 \\
(2,70 \%)\end{array}$ & $\begin{array}{l}3 \\
(2,03 \%)\end{array}$ & 148 \\
\hline 2010 & $\begin{array}{l}115 \\
(67,65 \%)\end{array}$ & $\begin{array}{l}31 \\
(18,24 \%)\end{array}$ & $\begin{array}{l}8 \\
(4,71 \%)\end{array}$ & $\begin{array}{l}8 \\
(4,71 \%)\end{array}$ & $\begin{array}{l}1 \\
(0,59 \%)\end{array}$ & $\begin{array}{l}4 \\
(2,35 \%)\end{array}$ & $\begin{array}{l}3 \\
(1,76 \%)\end{array}$ & 170 \\
\hline 2011 & $\begin{array}{l}121 \\
(68,75 \%)\end{array}$ & $\begin{array}{l}32 \\
(18,18 \%)\end{array}$ & $\begin{array}{l}7 \\
(3,98 \%)\end{array}$ & $\begin{array}{l}8 \\
(4,55 \%)\end{array}$ & $\begin{array}{l}1 \\
(0,57 \%)\end{array}$ & $\begin{array}{l}4 \\
(2,27 \%)\end{array}$ & $\begin{array}{l}3 \\
(1,70 \%)\end{array}$ & 176 \\
\hline
\end{tabular}

Fonte: elaborado pelos autores com base nos dados da pesquisa.

Observa-se o crescimento no número de cursos de graduação ofertados pelas instituições, com exceção da ULBRA, que diminuiu um curso em 2011, em comparação a 2010; e a FADISMA que foca sua atenção apenas na formação de bacharéis em Direito.

A Tabela 4 mostra o número de alunos matriculados nesses cursos de graduação. 
A influência de uma universidade na geração de um polo regional de ensino superior Moacir Luiz Vighi, Janis Elisa Ruppenthal

Tabela 4: Número de alunos matriculados nas instituições da cidade de Santa Maria

\begin{tabular}{|c|c|c|c|c|c|c|c|c|}
\hline Ano & UFSM & UNIFRA & ULBRA & FAMES & FADISMA & FAPAS & FISMA & TOTAL \\
\hline 1997 & $\begin{array}{c}10715 \\
(89,11 \%)\end{array}$ & $\begin{array}{c}1309 \\
(10,89 \%)\end{array}$ & & & & & & 12024 \\
\hline 1998 & $\begin{array}{c}11107 \\
(86,60 \%)\end{array}$ & $\begin{array}{c}1538 \\
(11,99 \%) \\
\end{array}$ & & $\begin{array}{c}180 \\
(1,40 \%)\end{array}$ & & & & 12825 \\
\hline 1999 & $\begin{array}{c}11874 \\
(86,86 \%)\end{array}$ & $\begin{array}{c}1556 \\
(11,38 \%)\end{array}$ & & $\begin{array}{c}240 \\
(1,76 \%)\end{array}$ & & & & 13670 \\
\hline 2000 & $\begin{array}{c}12523 \\
(80,44 \%)\end{array}$ & $\begin{array}{c}2746 \\
(17,64 \%)\end{array}$ & & $\begin{array}{c}300 \\
(1,93 \%)\end{array}$ & & & & 15569 \\
\hline 2001 & $\begin{array}{c}12839 \\
(76,38 \%)\end{array}$ & $\begin{array}{c}3621 \\
(21,54 \%) \\
\end{array}$ & & $\begin{array}{c}350 \\
(2,08 \%)\end{array}$ & & & & 16810 \\
\hline 2002 & $\begin{array}{c}13100 \\
(72,79 \%)\end{array}$ & $\begin{array}{c}4194 \\
(23,30 \%)\end{array}$ & $\begin{array}{c}300 \\
(1,67 \%)\end{array}$ & $\begin{array}{c}368 \\
(2,04 \%)\end{array}$ & & $\begin{array}{c}35 \\
(0,19 \%)\end{array}$ & & 17997 \\
\hline 2003 & $\begin{array}{c}13147 \\
(70,15 \%)\end{array}$ & $\begin{array}{c}4685 \\
(25,00 \%)\end{array}$ & $\begin{array}{c}400 \\
(2,13 \%)\end{array}$ & $\begin{array}{c}390 \\
(2,08 \%)\end{array}$ & & $\begin{array}{c}118 \\
(0,63 \%)\end{array}$ & & 18740 \\
\hline 2004 & $\begin{array}{c}13079 \\
(65,30 \%)\end{array}$ & $\begin{array}{c}5335 \\
(26,64 \%)\end{array}$ & $\begin{array}{c}500 \\
(2,50 \%) \\
\end{array}$ & $\begin{array}{c}621 \\
(3,10 \%) \\
\end{array}$ & $\begin{array}{c}200 \\
(1,00 \%) \\
\end{array}$ & $\begin{array}{c}154 \\
(0,77 \%) \\
\end{array}$ & $\begin{array}{c}139 \\
(0,69 \%) \\
\end{array}$ & 20028 \\
\hline 2005 & $\begin{array}{c}12882 \\
(60,56 \%)\end{array}$ & $\begin{array}{c}5856 \\
(27,53 \%)\end{array}$ & $\begin{array}{c}800 \\
(3,76 \%)\end{array}$ & $\begin{array}{c}855 \\
(4,02 \%)\end{array}$ & $\begin{array}{c}335 \\
(1,57 \%)\end{array}$ & $\begin{array}{c}250 \\
(1,18 \%)\end{array}$ & $\begin{array}{c}293 \\
(1,38 \%)\end{array}$ & 21271 \\
\hline 2006 & $\begin{array}{c}13043 \\
(57,70 \%) \\
\end{array}$ & $\begin{array}{c}6195 \\
(27,40 \%) \\
\end{array}$ & $\begin{array}{c}1000 \\
(4,42 \%) \\
\end{array}$ & $\begin{array}{c}1082 \\
(4,79 \%) \\
\end{array}$ & $\begin{array}{c}486 \\
(2,15 \%) \\
\end{array}$ & $\begin{array}{c}387 \\
(1,71 \%) \\
\end{array}$ & $\begin{array}{c}413 \\
(1,83 \%) \\
\end{array}$ & 22606 \\
\hline 2007 & $\begin{array}{c}13250 \\
(55,69 \%)\end{array}$ & $\begin{array}{c}6553 \\
(27,54 \%)\end{array}$ & $\begin{array}{c}1200 \\
(5,04 \%)\end{array}$ & $\begin{array}{c}1193 \\
(5,01 \%)\end{array}$ & $\begin{array}{c}522 \\
(2,19 \%)\end{array}$ & $\begin{array}{c}563 \\
(2,37 \%)\end{array}$ & $\begin{array}{c}511 \\
(2,15 \%)\end{array}$ & 23792 \\
\hline 2008 & $\begin{array}{c}13771 \\
(56,37 \%)\end{array}$ & $\begin{array}{c}6645 \\
(27,20 \%)\end{array}$ & $\begin{array}{c}1400 \\
(5,73 \%)\end{array}$ & $\begin{array}{c}963 \\
(3,94 \%)\end{array}$ & $\begin{array}{c}599 \\
(2,45 \%)\end{array}$ & $\begin{array}{c}614 \\
(2,51 \%)\end{array}$ & $\begin{array}{c}436 \\
(1,78 \%)\end{array}$ & 24428 \\
\hline 2009 & $\begin{array}{c}15511 \\
(59,78 \%)\end{array}$ & $\begin{array}{c}6584 \\
(25,37 \%)\end{array}$ & $\begin{array}{c}1000 \\
(3,85 \%)\end{array}$ & $\begin{array}{c}1183 \\
(4,56 \%)\end{array}$ & $\begin{array}{c}588 \\
(2,27 \%)\end{array}$ & $\begin{array}{c}687 \\
(2,65 \%)\end{array}$ & $\begin{array}{c}394 \\
(1,52 \%)\end{array}$ & 25947 \\
\hline 2010 & $\begin{array}{c}17134 \\
(63,08 \%)\end{array}$ & $\begin{array}{c}6275 \\
(23,10 \%)\end{array}$ & $\begin{array}{c}1000 \\
(3,68 \%)\end{array}$ & $\begin{array}{c}963 \\
(3,55 \%)\end{array}$ & $\begin{array}{c}598 \\
(2,20 \%)\end{array}$ & $\begin{array}{c}802 \\
(2,95 \%)\end{array}$ & $\begin{array}{c}389 \\
(1,43 \%)\end{array}$ & 27161 \\
\hline 2011 & $\begin{array}{c}18649 \\
(64,97 \%)\end{array}$ & $\begin{array}{c}6289 \\
(21,91 \%)\end{array}$ & $\begin{array}{c}900 \\
(3,14 \%)\end{array}$ & $\begin{array}{c}852 \\
(2,97 \%)\end{array}$ & $\begin{array}{c}546 \\
(1,90 \%)\end{array}$ & $\begin{array}{c}915 \\
(3,19 \%)\end{array}$ & $\begin{array}{c}554 \\
(1,93 \%)\end{array}$ & 28705 \\
\hline
\end{tabular}

Fonte: elaborado pelos autores com base nos dados da pesquisa.

Também se observa na Tabela 4, um incremento no acesso ao ensino superior na cidade. Comparando-se o ano de 2004, ano em que todas as instituições possuem dados completos, com o ano de 2011, o número de alunos matriculados passou de 20028 para 28705 , o que significa um aumento de 43,32\%.

Já o número de docentes nas instituições de ensino superior da cidade de Santa Maria é apresentado na Tabela 5. 
Tabela 5: Número de docentes nas instituições da cidade de Santa Maria

\begin{tabular}{|c|c|c|c|c|c|c|c|c|}
\hline Ano & UFSM & UNIFRA & ULBRA & FAMES & FADISMA & FAPAS & FISMA & TOTAL \\
\hline 1997 & $\begin{array}{c}1440 \\
(94,12 \%)\end{array}$ & $\begin{array}{c}90 \\
(5,88 \%)\end{array}$ & & & & & & 1530 \\
\hline 1998 & $\begin{array}{c}1444 \\
(91,92 \%) \\
\end{array}$ & $\begin{array}{c}115 \\
(7,32 \%) \\
\end{array}$ & & $\begin{array}{c}12 \\
(0,76 \%)\end{array}$ & & & & 1571 \\
\hline 1999 & $\begin{array}{c}1420 \\
(82,22 \%)\end{array}$ & $\begin{array}{c}295 \\
(17,08 \%)\end{array}$ & & $\begin{array}{c}12 \\
(0,69 \%)\end{array}$ & & & & 1727 \\
\hline 2000 & $\begin{array}{c}1447 \\
(82,17 \%)\end{array}$ & $\begin{array}{c}300 \\
(17,04 \%)\end{array}$ & & $\begin{array}{c}14 \\
(0,80 \%)\end{array}$ & & & & 1761 \\
\hline 2001 & $\begin{array}{c}1417 \\
(80,15 \%)\end{array}$ & $\begin{array}{c}320 \\
(18,10 \%)\end{array}$ & & $\begin{array}{c}14 \\
(0,79 \%)\end{array}$ & & $\begin{array}{c}17 \\
(0,96 \%)\end{array}$ & & 1768 \\
\hline 2002 & $\begin{array}{c}1303 \\
(73,91 \%)\end{array}$ & $\begin{array}{c}389 \\
(22,06 \%)\end{array}$ & $\begin{array}{c}30 \\
(1,70 \%)\end{array}$ & $\begin{array}{c}16 \\
(0,91 \%)\end{array}$ & & $\begin{array}{c}25 \\
(1,42 \%)\end{array}$ & & 1763 \\
\hline 2003 & $\begin{array}{c}1350 \\
(76,06 \%)\end{array}$ & $\begin{array}{c}335 \\
(18,87 \%)\end{array}$ & $\begin{array}{c}40 \\
(2,25 \%)\end{array}$ & $\begin{array}{c}25 \\
(1,41 \%)\end{array}$ & & $\begin{array}{c}25 \\
(1,41 \%)\end{array}$ & & 1775 \\
\hline 2004 & $\begin{array}{c}1210 \\
(73,24 \%)\end{array}$ & $\begin{array}{c}340 \\
(20,58 \%)\end{array}$ & $\begin{array}{c}40 \\
(2,42 \%)\end{array}$ & $\begin{array}{c}28 \\
(1,69 \%)\end{array}$ & $\begin{array}{c}6 \\
(0,36 \%) \\
\end{array}$ & $\begin{array}{c}28 \\
(1,69 \%) \\
\end{array}$ & & 1652 \\
\hline 2005 & $\begin{array}{c}1284 \\
(72,62 \%)\end{array}$ & $\begin{array}{c}344 \\
(19,46 \%)\end{array}$ & $\begin{array}{c}50 \\
(2,83 \%)\end{array}$ & $\begin{array}{c}30 \\
(1,70 \%)\end{array}$ & $20(1,13 \%)$ & $\begin{array}{c}40 \\
(2,26 \%)\end{array}$ & & 1768 \\
\hline 2006 & $\begin{array}{c}1311 \\
(70,64 \%)\end{array}$ & $\begin{array}{c}390 \\
(21,01 \%)\end{array}$ & $\begin{array}{c}60 \\
(3,23 \%)\end{array}$ & $\begin{array}{c}32 \\
(1,72 \%)\end{array}$ & $19(1,02 \%)$ & $\begin{array}{c}44 \\
(2,37 \%)\end{array}$ & & 1856 \\
\hline 2007 & $\begin{array}{c}1298 \\
(68,53 \%)\end{array}$ & $\begin{array}{c}396 \\
(20,91 \%)\end{array}$ & $\begin{array}{c}60 \\
(3,17 \%)\end{array}$ & $\begin{array}{c}34 \\
(1,80 \%)\end{array}$ & $53(2,80 \%)$ & $\begin{array}{c}53 \\
(2,80 \%)\end{array}$ & & 1894 \\
\hline 2008 & $\begin{array}{c}1311 \\
(65,62 \%)\end{array}$ & $\begin{array}{c}396 \\
(19,82 \%)\end{array}$ & $\begin{array}{c}70 \\
(3,50 \%)\end{array}$ & $\begin{array}{c}40 \\
(2,00 \%)\end{array}$ & $39(1,95 \%)$ & $\begin{array}{c}52 \\
(2,60 \%)\end{array}$ & $\begin{array}{c}90 \\
(4,50 \% \\
)\end{array}$ & 1998 \\
\hline 2009 & $\begin{array}{c}1401 \\
(66,49 \%)\end{array}$ & $\begin{array}{c}396 \\
(18,79 \%)\end{array}$ & $\begin{array}{c}70 \\
(3,32 \%)\end{array}$ & $\begin{array}{c}42 \\
(1,99 \%)\end{array}$ & $51(2,42 \%)$ & $\begin{array}{c}49 \\
(2,33 \%)\end{array}$ & $\begin{array}{c}98 \\
(4,65 \% \\
)\end{array}$ & 2107 \\
\hline 2010 & $\begin{array}{c}1461 \\
(68,21 \%)\end{array}$ & $\begin{array}{c}398 \\
(18,58 \%)\end{array}$ & $\begin{array}{c}60 \\
(2,80 \%)\end{array}$ & $\begin{array}{c}45 \\
(2,10 \%)\end{array}$ & $28(1,31 \%)$ & $\begin{array}{c}55 \\
(2,57 \%)\end{array}$ & $\begin{array}{c}95 \\
(4,44 \% \\
)\end{array}$ & 2142 \\
\hline 2011 & $\begin{array}{c}1515 \\
(68,77 \%)\end{array}$ & $\begin{array}{c}402 \\
(18,25 \%)\end{array}$ & $\begin{array}{c}60 \\
(2,72 \%)\end{array}$ & $\begin{array}{c}47 \\
(2,13 \%)\end{array}$ & $25(1,13 \%)$ & $\begin{array}{c}58 \\
(2,63 \%)\end{array}$ & $\begin{array}{c}96 \\
(4,36 \%\end{array}$ & 2203 \\
\hline
\end{tabular}

Fonte: elaborado pelos autores com base nos dados da pesquisa.

O número de docentes acompanhou a evolução do número de cursos de graduação ofertados e do número de alunos matriculados nas instituições de ensino superior de Santa Maria, alcançando em 2011 o número de 2203 docentes. 


\subsection{Análise estatística dos dados}

Essa análise foi realizada comparando-se a UFSM com cada uma das demais instituições de ensino superior com curso presencial em Santa Maria, através de análise não paramétrica. A Tabela 6 , elaborada com base nos dados obtidos do software "Statistica 9.0 ®", mostra os dados da análise estatística em relação ao número de cursos de graduação presenciais.

Tabela 6: Análise estatística dos cursos de graduação nas instituições de Santa Maria

\begin{tabular}{|c|c|c|c|}
\hline \multirow{2}{*}{ Instituições } & \multirow{2}{*}{$\begin{array}{c}\text { Número de } \\
\text { comparações (N) }\end{array}$} & \multicolumn{2}{|c|}{ Correlações não paramétricas } \\
\cline { 3 - 4 } & 15 & Spearman (R) & (p-level) \\
\hline UFSM x UNIFRA & 10 & 0,944927 & $<0,0001$ \\
\hline UFSM x ULBRA & 14 & 0,692952 & 0,026311 \\
\hline UFSM x FAMES & 8 & - & 0,00022 \\
\hline UFSM x FADISMA & 12 & 0,884652 & - \\
\hline UFSM x FAPAS & 8 & 0,755929 & 0,000132 \\
\hline UFSM x FISMA & & &
\end{tabular}

Fonte: elaborado pelos autores com base nos dados da pesquisa.

Analisando-se os dados da correlação não paramétrica, evidencia-se uma correlação entre a UFSM e todas as instituições de Santa Maria, com $p<0,05$ em todas as análises, variando de 0 ao valor máximo de 0,030020.

A Tabela 7 apresenta os dados obtidos do software "Statistica $9.0 \AA$ ", para as variáveis relacionadas com o número de alunos presenciais de graduação.

Tabela 7: Análise estatística dos alunos de graduação nas instituições de Santa Maria

\begin{tabular}{|c|c|c|c|}
\hline \multirow{2}{*}{ Instituições } & \multirow{2}{*}{$\begin{array}{c}\text { Número de } \\
\text { comparações }(\mathbf{N})\end{array}$} & \multicolumn{2}{|c|}{ Correlações não paramétricas } \\
\cline { 3 - 4 } & 15 & 0,878571 & 0,00016 \\
\hline UFSM x UNIFRA & 10 & 0,386532 & 0,269869 \\
\hline UFSM x ULBRA & 14 & 0,715072 & 0,004045 \\
\hline UFSM x FAMES & 8 & 0,714286 & 0,046528 \\
\hline UFSM x FADISMA & 10 & 0,781818 & 0,007547 \\
\hline UFSM x FAPAS & 8 & 0,476190 & 0,232936 \\
\hline UFSM x FISMA & & &
\end{tabular}

Fonte: elaborado pelos autores com base nos dados da pesquisa. 
Da mesma forma, analisando-se as correlações não paramétricas, a UFSM possui correlação positiva com quatro instituições - UNIFRA, FAMES, FADISMA e FAPAS, com $p$ variando de 0,000016 a 0,046528.

$\mathrm{Na}$ Tabela 8 são mostrados os dados estatísticos das análises comparativas entre o número de professores de graduação das instituições.

Tabela 8: Análise estatística dos docentes de graduação nas instituições de Santa Maria

\begin{tabular}{|c|c|c|c|}
\hline \multirow{2}{*}{ Instituições } & \multirow{2}{*}{$\begin{array}{c}\text { Número de } \\
\text { comparações (N) }\end{array}$} & \multicolumn{2}{|c|}{ Correlações não paramétricas } \\
\cline { 3 - 4 } & 15 & $-0,082512$ & 0,770026 \\
\hline UFSM x UNIFRA & 10 & 0,460845 & 0,180097 \\
\hline UFSM x ULBRA & 14 & 0,015436 & 0,958232 \\
\hline UFSM x FAMES & 8 & 0,335335 & 0,416792 \\
\hline UFSM x FADISMA & 11 & 0,324201 & 0,330725 \\
\hline UFSM x FAPAS & 4 & 0,400000 & 0,600000 \\
\hline UFSM x FISMA & & &
\end{tabular}

Fonte: elaborado pelos autores com base nos dados da pesquisa.

Observa-se que não existe correlação, em nenhum momento, para essa variável. Isso pode ser explicado pelo fato de o Brasil possuir 10,64 alunos de nível superior por professor (RIGHI; RUPPENTHAL, 2012). Além disso, a abertura de cursos de áreas correlatas faz com que não seja necessária a contratação de novos professores, mas sim o aumento de carga horária dos docentes já contratados.

\section{CONSIDERAÇÕES FINAIS}

O objetivo desse artigo foi avaliar a contribuição da UFSM na criação de um polo de ensino superior em Santa Maria. Pode ser observado que a instalação das instituições de ensino superior após a criação da UFSM deu-se, na maioria dos casos, pelo fato do surgimento de novas áreas de ensino e de novas tecnologias, além dessas instituições considerarem a cidade um polo de ensino superior no estado. Com o surgimento desse polo de ensino superior em Santa Maria, observou-se o incremento 
no número de cursos de graduação. Com a oferta crescente, o incentivo governamental e a diversidade de cursos ofertados, o número de alunos também cresceu nos últimos quinze anos.

Com esse crescimento, a pesquisa detectou o importante aumento no número de pessoas diretamente envolvidas no ensino superior em Santa Maria. O número de alunos nos cursos presenciais de graduação cresceu 138,73\%, passando de 12024 para 28705, em quinze anos. Já o número de professores passou de 1530 para 2203, representando um aumento de 43,99\%.

Através da análise estatística dos dados pesquisados, estabeleceu-se a correlação das variáveis analisadas da Universidade Federal de Santa Maria com as demais instituições. Isso determinou uma correlação positiva entre o crescimento do número de cursos presenciais de graduação e do número de alunos das instituições em relação à UFSM. Já o número de professores não apresentou correlação entre as instituições.

Assim, confirmou-se a hipótese desse artigo, que era de que a UFSM contribuiu para o desenvolvimento de um polo de ensino superior na cidade de Santa Maria, o que foi demonstrado pela existência de correlações positivas entre os números de cursos e de alunos em cursos de graduação presenciais em Santa Maria. Salienta-se que essa correlação existe, embora haja algumas limitações nessa pesquisa, ou seja, os levantamentos foram realizados apenas nas instituições de ensino superior com cursos presenciais e somente considerando os cursos de graduação.

\section{REFERÊNCIAS}

BASTOS, R.C. (1987). Sistemas de Custos para as Universidades Federais Autárquicas. Revista de Administração Pública, 21(3):58-81.

BOVO, J.M. (2008). Impactos Econômicos e Financeiros da UNESP Para os Municípios. São Paulo, UNESP. 
DRUCKER, J., GOLDSTEIN, H. (2007). Assessing the Regional Economic Development Impacts of Universities: a review of current approaches. International Regional Science Review, 30(1):20-46.

FAVA-DE-MORAES, F. (2000). Universidade, inovação e impacto socioeconômico. São Paulo em Perspectiva, 14(3):8-11.

FELSENSTEIN, D. (1996). The University in the Metropolitan Arena: Impacts and Public Policy Implications. Urban Studies, 33(9):1565-1580.

GARRIDO-YSERTE, R.; GALLO-RIVERA, M.T. (2010). O impacto da universidade sob a economia local: três métodos para estimar os efeitos colaterais da demanda. The Annals of Regional Science, 44(1):39-67.

GOLDSTEIN, H.A.; GLASER, K. (2012). Research universities as actors in the governance of local and regional development. The Journal of Technology Transfer, $37(2): 158-174$.

GOLDSTEIN, H.A.; RENAULT, C.S. (2005). Estimating Universities Contributions to Regional Economic Development: The Case of the U.S., Spillovers and Innovations. New York, Springer.

GUBIANI, J.S.; MORALES, A.B.T.; SELIG, P.M.; LOPES, L.F.D. (2010). Atividades das universidades e o impacto no desenvolvimento regional. In: XXX Encontro Nacional de Engenharia de Produção, São Carlos, 2010. Anais... São Carlos, UFSCar.

HOFF, D.N., MARTIN, A.S.S., SOPEÑA, M.B. (2011). Universidades e desenvolvimento regional: impactos quantitativos da Unipampa em Sant'ana do Livramento. Redes, 16(3):157-183.

LENDEL, I. (2010). The impact of research universities on regional economies: the concept of university products. Economic Development Quarterly, 24(3):210-230.

MALHOTRA. N.K. (2006). Pesquisa de Marketing: uma orientação aplicada. 4. ed. Porto Alegre, Bookman.

MARINHO, A. (1996). Avaliação organizacional de uma universidade pública: uma abordagem não paramétrica da Universidade Federal do Rio de Janeiro. Rio de Janeiro, RJ. Tese de Doutorado. Fundação Getúlio Vargas - FGV, Rio de Janeiro.

RIGHI, M.L.; RUPPENTHAL, J.E. (2012). Importância do ensino superior nas ações empreendedoras. Rev. CCEI - URCAMP, 16(29):190-205. 
ROLIM, C.; KURESKI, R. (2007). Impacto Econômico de Curto Prazo das Universidades Estaduais Paranaenses-2004. Revista Paranaense de Desenvolvimento, v. 112, p. 111-130.

ROLIM, C.; SERRA, M. (2009). Instituições de ensino superior e desenvolvimento regional: o caso da região Norte do Paraná. Revista de Economia, 35(3):87-102.

SCHWARTZMAN, J. (1995). Dificuldades e possibilidades de se construir um ranking para as universidades brasileiras. Ensaio, 3(6):5-28.

SIEGFRIED, J.J.; SANDERSON, A.R.; MCHENRY, P. (2007). The economic impact of colleges and universities. Economics of Education Review, 26(5):546-558.

STEINACKER, A. (2005). The Economic Effect of Urban Colleges on their Surrounding Communities. Urban Studies, 42(7):1161-1175.

WARREN, A.; HANKE, R.; TROTZER, D. (2008). Models for university technology transfer: resolving conflicts between mission and methods and the dependency on geographic location. Cambridge Journal of Regions, Economy and Society, 1(2):219232.

Data de Submissão: 24/09/2012.

Data de Aceite: 14/03/2013. 